\title{
ESTUDO DE UM SISTEMA OSCILANTE COM MASSA VARIÁVEL
}

\author{
Fabiana Castro ${ }^{1}$, Paulo S. Tomé ${ }^{1}$, G. Silva Neto ${ }^{1}$, J. S. Espinoz Ortiz ${ }^{1}$ \\ 1. Departamento de Fśisca \\ Univerisidade Federal de Goiás, \\ Catalã, GO 75704020, Brasil
E-mails: fabicastro19@gmail.com, paulosergio.fisica.ps@gmail.com, gilmarneto@gmail.com, jsespinophysics@gmail.com

\begin{abstract}
In this work we studied an harmonic oscillator consisting of a plastic bottle filled with sand and which is suspended by a spring. The system loses mass through the flow of a granular material, which was need to be sifted to standardize the grains. We used two plastic covers with regular transverse sections, as to generate different rates of flow. The acquisition of experimental data measurements were performed by using: A sensor force, a sensor position and a interface to a computer. We were able to measure the flow rate of mass by using the force sensor, and with the position sensor we measured the instant position of the dynamical system. To describe theoretically the dynamic of the system, our analysis took its starting point the Newton's second law. We verify the presence of two types of movements: A translational movement of the equilibrium point and a purely oscillatory movement, which together constitute a movement composed of two normal modes. Thus we conclude that the variable mass harmonic oscillator is a good system to work the Newton's equation in its fullness.
\end{abstract}

\begin{abstract}
Keywords_ - Harmonic oscillator, Variable mass, Newton's second law.
Resumo- Neste trabalho estudamos um oscilador harmônico que consiste de uma garrafa plástica que armazena areia e que é pendurada por uma mola. O sistema perde massa através do escoamento do material granular, que precisou ser peneirada a fim de uniformizar os grãos. Utilizamos duas tampas plásticas com área de seção transversal regular, como para gerar diferentes taxas de escoamento. A aquisição de medidas experimentais foi executada utilizando: Um sensor de força, um sensor de posição e uma interfase com o computador. Com o sensor de força fomos capazes de medir a taxa de escoamento de massa, já com o sensor de posição medimos a posição instantânea do sistema dinâmico. Para descrever teoricamente a dinâmica do sistema, nossa análise teve como seu ponto de partida a segunda lei de Newton. Verificamos dos tipos de movimento presentes: O movimento de translação do ponto de equilíbrio e o movimento puramente oscilatório, que em conjunto constituem um movimento composto por dois modos normais. Concluímos então que o oscilador com massa variável é um bom sistema para se trabalhar as equações de Newton na sua plenitude.
\end{abstract}

Palavras-chave— Oscilador harmônico, massa variável, Segunda lei de Newton.

\section{Introdução}

O oscilador harmônico simples (OHS) é um dos sistemas mais empregados na modelagem da Física [1-2]. Devido a sua simplicidade e fácil descrição pelas equações de Newton na mecânica clássica, tal sistema descreve perfeitamente o balanço energético entre energia potencial elástica e energia cinética. $\mathrm{Na}$ mecânica quântica é o principal protagonista das hipóteses de quantização com resultados empíricos fantásticos. Como sistema mecânico é um excelente sistema para o estudo e a modelagem para movimentos periódicos.

Dentre as diferentes formas de OHS estamos interessados no oscilador harmônico pendurado por uma mola no qual a força peso está diretamente associada com a posição de equilíbrio em repouso. Além disso, queremos fazer com que o sistema perca massa através do escoamento de um material granular, especificamente areia. O esquema experimental é mostrado na Figura 1 onde são representados, à esquerda, a montagem do aparato experimental para medir o escoamento de massa, assim como a montagem experimental requerida para realizar as medidas de posição, à direita. Partiremos de um modelo teórico preestabelecido no estudo desse sistema [3] para descrevermos meticulosamente os dados experimentais obtidos. O estudo de sistemas com a variação de certos parâmetros constitui-se um bom aprofundamento no que tange as famosas leis que mudaram o mundo no século XVII, as leis de Newton. Mostraremos que o movimento resultante será composto por dois modos normais: um de translação e outro de oscilação através da segunda lei de Newton.

\section{Marco Teórico}

Como no problema de perda de massa em um foguete [5], nosso ponto de partida será a variação de momento após a pequena porção de massa $\Delta m$ deixar a garrafa. Definindo $q=\dot{y}_{2}-\dot{y}_{1}$ como a velocidade relativa da porção de massa $\Delta m$ com relação à garrafa. Onde $y_{1}$ e $y_{2}$ são as distância entre o ponto de fixação à extremidade do suporte do peso e a distância entre o ponto de fixação à pequena porção de massa $\Delta m$ que sai da garrafa, respectivamente, Figura 1. Portanto, se considerarmos certo instante de 
tempo $t$ e um pequeno intervalo de tempo $\Delta t$ imediatamente posterior, podemos escrever, no limite $\Delta t \rightarrow 0$ :

$$
\dot{P}=m(t) \ddot{y}_{1}-q \dot{m}(t)
$$

Onde o segundo termo dessa equação é responsável pelo impulso devido a ejeção da massa, como o recuo de uma arma ao ser disparada. Contudo, devido à ejeção se dar apenas pela força da gravidade, e ademais, considerando-se o efeito da granularidade da areia, podemos afirmar que a pequena quantidade de impulso assim a pequena porção de massa deixa a garrafa é desprezível em relação à grande quantidade de momento da garrafa. Consequentemente, a segunda lei de Newton para a garrafa pode ser escrita como:

$$
m(t) \ddot{y} \approx-\mathrm{\kappa} y+\mathrm{\kappa}(h-\delta)-b \dot{y}+m(t) g \text {. }
$$

Onde $b$ é a resistência do ar, $\kappa$ a constante da mola e $\Delta=\ell_{0}+\delta$. Foi feita a mudança de variáveis $y_{1} \rightarrow h-y$. A variável $y$ é medida a partir do sensor, como mostrado na figura a baixo.

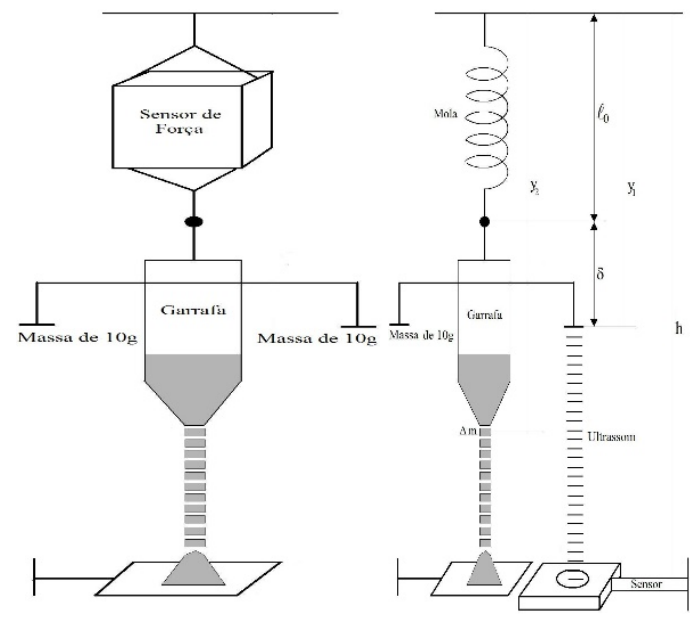

Figura 1. Diferentes esquemas de montagem do aparato experimental utilizado para medir o escoamento de massa e da posição, à esquerda e à direita da figura, respectivamente.

Neste ponto, somos fortemente tentados a escrever para $y(t)=y_{p}(t)+y_{\text {osc }}(t)$, assim separando o movimento da garrafa numa combinação de dois tipos de movimento: um em relação ao deslocamento do ponto de equilíbrio $y_{p}(t)$, e o último relacionado a um movimento puramente oscilatório descrito por $y_{\text {osc }}(t)$ :

\section{Modelagem e análise da pesquisa}

Para a aquisição de medidas utilizamos os sensores disponíveis pela CIDEPE. Um sensor de força, um sensor de posição e a interface com o computador chamado Lab100. A primeira aquisição se refere ao escoamento de massa montado segundo o lado esquerdo na Figura 1. Para isso foram utilizadas duas tampas de uma garrafa plástica média com área de seção transversal regular, com orifício circular de diâmetros 5,20 $\mathrm{mm}$ e $7,73 \mathrm{~mm}$. Na garrafa para efeitos de medidas da posição, foi transpassado perpendicularmente à linha de centro de massa um arame grosso para se pendurar dois suportes de pequenas massas de $10 \mathrm{~g}$ que, além de adicionar massa própria, servem de superfície refletora para o sensor de posição, como mostrado à direita da mesma figura. Aqui devemos mencionar as grandezas físicas importantes com a quais foram obtidos os dados experimentais. Para o comprimento inicial da mola $\ell_{o} \approx 0,115 m$, já para a distância entre as extremidades de um dos pesos e o ponto de junção mola garrafa $\delta \approx 0,182 \mathrm{~m}$. Enquanto que a distância do sensor até o ponto de fixação da mola-garrafa estabelecemos $h \approx 1,196 m$. Finalmente, a constante da mola utilizada $\kappa=6,5 \mathrm{~N} / \mathrm{m}$.

O comportamento do escoamento da areia ao longo do tempo, mantendo a garrafa em repouso, é mostrado na Figura 2. Encontramos que o comportamento é linear, $m(t)=m_{0}-c t$, com $c_{1}=0,0021 \mathrm{~kg} / \mathrm{s}$ e $c_{2}=0,0063 \mathrm{~kg} / \mathrm{s}$ para orifícios com menor e maior diâmetro, respectivamente. Para um material granular de densidade $\rho$ que flui, por causa ação gravitacional, através de um orifício de diâmetro $D$, a taxa de escoamento satisfaz a fórmula de Yersel [4]:

$$
c=-\dot{m}=\text { const } \times \rho g^{1 / 2}(D-d)^{5 / 2},
$$

sendo $d$ o diâmetro médio do grão de areia que no nosso caso é de aproximadamente $1,0 \mathrm{~mm}$.

Os valores antes mencionados foram satisfatoriamente computados através desta formulação.

$$
\begin{aligned}
& y_{p}(t)=(h-\Delta)-\frac{m_{0} g}{\mathrm{~K}}-\frac{c b g}{\mathrm{~K}^{2}}+\frac{c g}{\mathrm{~K}} t, \\
& 0=m(t) \frac{d^{2} y_{o s c}}{d t^{2}}+b \frac{d y_{o s c}}{d t}+\mathrm{K} y_{o s c} .
\end{aligned}
$$




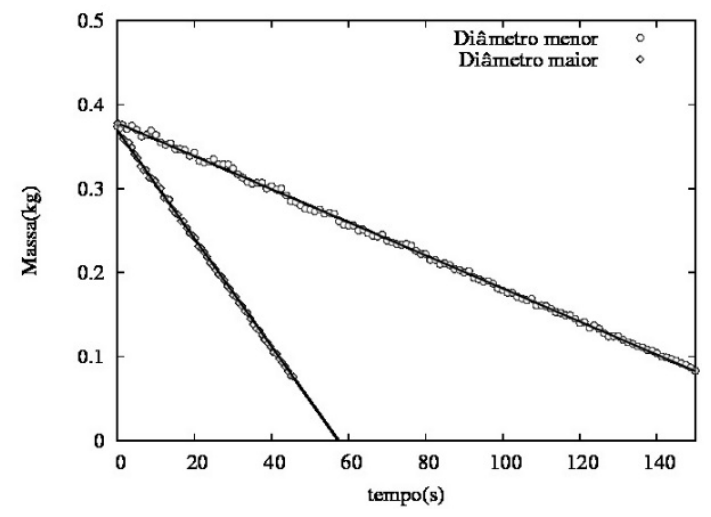

Figura 2. O escoamento da massa em função do tempo para as tampas com furos de diâmetro menor $(5,20 \mathrm{~mm})$ e diâmetro maior $(7,73$ $\mathrm{mm})$, respectivamente.

O coeficiente de amortecimento é determinado na Figura 3, onde mostramos a amplitude em função do tempo para o movimento oscilatório da garrafa, sem escoamento de massa. O ajuste na escala logarítmica no eixo vertical determinou que $\gamma=b / 2 \mathrm{~m}_{0} \approx 0,0274 s^{-1}$. Na Figura 4 mostramos os dados experimentais assim como o ajuste de curva para o deslocamento da garrafa em função do

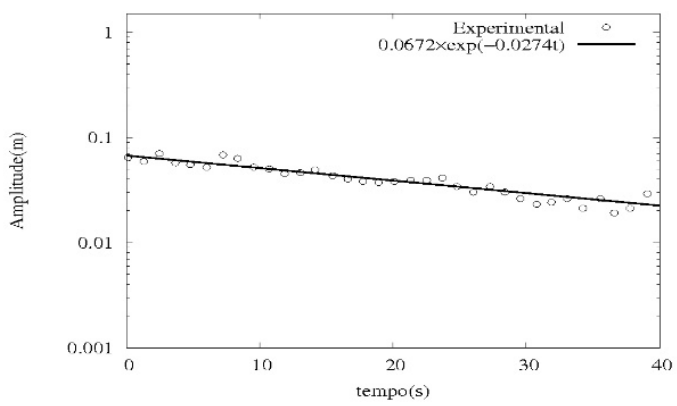

Figura 3. Determinação do coeficiente de amortecimento, através da medida da amplitude em função do tempo para o movimento oscilatório amortecido sem escoamento.

Figura 4. Variação temporal da posição da garrafa para o escoa-

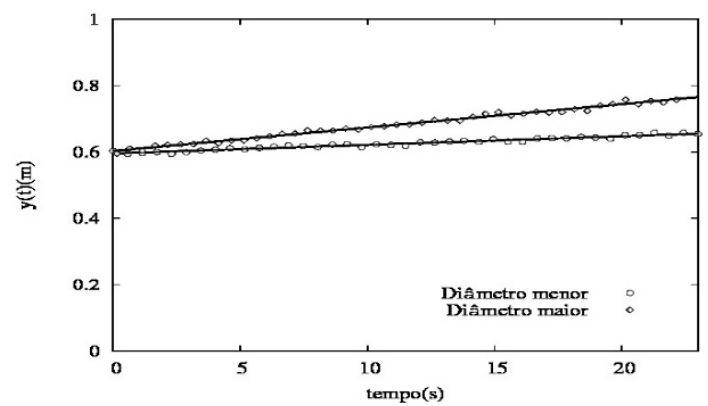

mento partindo-se do repouso, na posição de equilíbrio, para as tampas com os mesmos furos a cima.

do tempo, tendo como posição inicial a posição de equilíbrio. Para os diferentes diâmetros das tampas, encontramos que o escoamento é aproximadamente linear. Maior o diâmetro da tampa maior a pendente da curva.
Figura 5. A posição da garrafa em função do tempo para o movi-

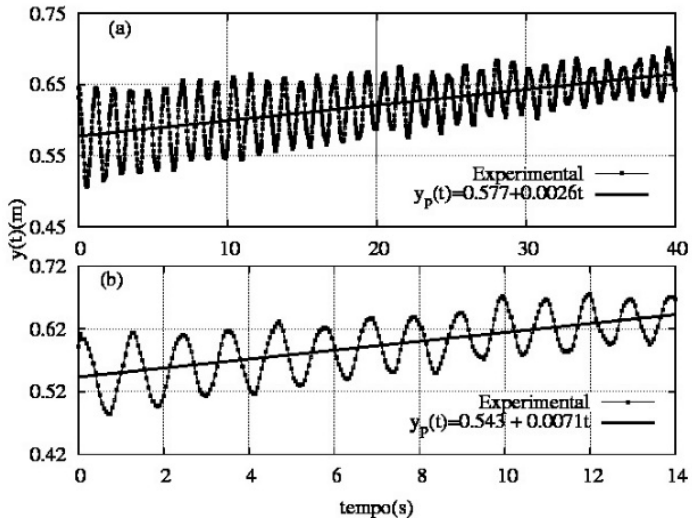

mento com escoamento partindo-se do repouso, fora da posição de equilíbrio. A figura superior refere-se à tampa menor e à inferior a tampa maior.

A Figura 5 mostra as medidas da posição oscilante da garrafa em função do tempo considerando os diferentes orifícios. $y_{p}(t)$ é determinado via regressão linear, note-se os diferentes valores para $y_{p}(0)$. Seguidamente, na Figura 6 apresentamos o movimento puramente oscilatório onde foi subtraído o modo de translação do ponto de equilíbrio.

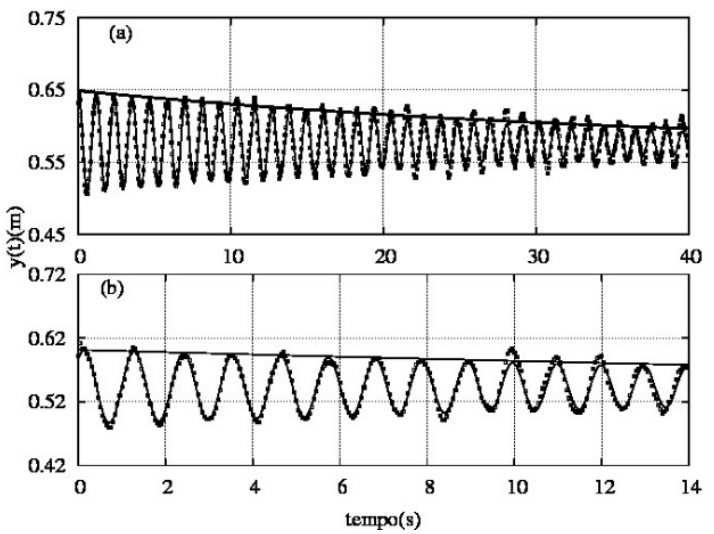

Figura 6. O movimento puramente oscilatório da garrafa, onde foi subtraído o modo de translação do ponto de equilíbrio (Figura 4); para a garrafa com tampa menor e maior, respectivamente.

\section{Conclusões}

Neste trabalho realizamos inúmeras medidas experimentais a fim de confirmar várias previsões teóricas e experimentais disponíveis na literatura $[3,4]$. Para analisar o escoamento granular utilizamos a fórmula empírica de Yersel [4] para orifícios circulares, verificado a partir das medidas com o sensor de força. Em seguida, partindo-se do repouso e de uma posição fora do equilíbrio inicial medimos as oscilações do sistema com massa variável. Visando descrever o movimento da garrafa, nossa análise tem como ponto de partida a segunda lei de Newton. Verificam-se dois tipos de movimento presentes: O movimento de translação do ponto de equilíbrio e o movimento puramente oscilatório, constitui um movimento composto por dois modos normais. Concluímos então que o oscilador com 
massa variável é um bom sistema para se trabalhar as equações de Newton na sua plenitude.

\section{Agradecimentos}

Os autores agradecem ao Dr. Eberth Correia pelas excelentes discussões durante a execução deste trabalho.

\section{Referências}

[1] B.E. Palladino e P. Leal Ferreira, Revista Brasileira de Ensino de Física 21, 490 (1999).
[2] A.C. Bertuola, M.S. Hussein e M.P. Pato, Revista Brasileira de Ensino de Física 27, 327 (2005).

[3] J. Flores, G. Solovey e S. Gil, Am. J. Phys. 71 (7), 721 (2003).

[4] M. Yersel, Phys. Teach. 38, 290 (2000).

[5] D. Halliday, R. Resnick e J. Walker, "Fundamentals of Physics" 8th edition (2010). 